\title{
A scoring system combining clinical, radiological, and histopathological examinations for differential diagnosis between lipoma and atypical lipomatous tumor/well-differentiated liposarcoma
}

\section{Yohei Asano}

Kanazawa University Graduate School of Medical Science

\section{Shinji Miwa}

Kanazawa University Graduate School of Medical Science Norio Yamamoto ( $\nabla$ norinori@med.kanazawa-u.ac.jp )

Kanazawa University Graduate School of Medical Science Katsuhiro Hayashi

Kanazawa University Graduate School of Medical Science Akihiko Takeuchi

Kanazawa University Graduate School of Medical Science

Kentaro Igarashi

Kanazawa University Graduate School of Medical Science

Hirotaka Yonezawa

Kanazawa University Graduate School of Medical Science

Yoshihiro Araki

Kanazawa University Graduate School of Medical Science

\section{Sei Morinaga}

Kanazawa University Graduate School of Medical Science

\section{Takayuki Nojima}

Kanazawa University Graduate School of Medical Science

Hiroko Ikeda

Kanazawa University Hospital

Hiroyuki Tsuchiya

Kanazawa University Graduate School of Medical Science

\section{Research Article}

Keywords: adipocytic tumor, atypical lipomatous tumor, well-differentiated liposarcoma, lipoma

Posted Date: March 17th, 2021 
DOl: https://doi.org/10.21203/rs.3.rs-279121/v1

License: (c) (1) This work is licensed under a Creative Commons Attribution 4.0 International License. Read Full License 


\section{Abstract}

Background: Preoperative differential diagnosis between atypical lipomatous tumor (ALT)/welldifferentiated liposarcoma (WDLS) and lipoma is important to determine the appropriate resection margin. However, since their radiological and histopathological findings are similar, it may be difficult to make a differential diagnosis. In this study, we evaluated the diagnostic accuracies of clinical, radiological, and histopathological examinations for diagnosing ALT/WDLS and developed a new combined scoring system.

Methods: Eighty-nine ALT/WDLS and 56 lipomas were included and their clinical characteristics, magnetic resonance imaging (MRI) findings, histological findings by hematoxylin and eosin (HE) staining were investigated, and compared between ALT/WDLS and lipoma. Then, univariate analyses and multivariate logistic regression analyses were performed for the findings, and a combined scoring system consisted of predictive factors of ALT/WDLS was developed.

Results: The univariate and multivariate logistic regression analyses revealed that tumor location (lower extremity), deep site, and size $(>11 \mathrm{~cm})$, thick septa $(>2 \mathrm{~mm})$, enhancement of septa or nodular lesions on $\mathrm{MRI}$, and lipoblasts in HE staining were significantly different for the diagnosis of ALT/WDLS. Based on the six predictive factors, we developed a combined scoring system (total 0-16 points, the cutoff was 9 points). The mean score of ALT/WDLS was significantly higher than lipoma (11.9 vs. 5.6, $p<0.0001)$. The area under the curve was 0.945 , and sensitivity was $87.6 \%$ and specificity was $91.1 \%$ by the receiver operating characteristics curve.

Conclusions: We developed a new combined scoring system based on comprehensive examinations that excluded examination which requires special equipment and reagents such as fluorescence in situ hybridization (FISH), and this can be used in many medical institutions. The diagnostic accuracy of ALT/WDLS was high and it may be useful for preoperative diagnosis and surgical plan.

\section{Introduction}

Adipocytic tumors are the most common soft tissue tumors, and the frequency of lipoma and atypical lipomatous tumor (ALT)/well-differentiated liposarcoma (WDLS) is high in clinical practice [1]. Most of the adipocytic tumors are either lipomas or ALTs, accounting for $40-45 \%$ of all tumors of the adipose tissue [2, 3]. On the other hand, liposarcomas are the most common soft tissue sarcoma [4]. ALT/WDLS is the most common subtype of liposarcoma, accounting for $40-45 \%$ of all liposarcomas $[5,6]$. It has been reported that ALT and WDLS are essentially synonymous, as the lesions are morphologically and karyotypically identical distinguished only by the tumor site. Adipocytic tumors located in the retroperitoneal or regions in which the tumor cannot be resected with a sufficient margin are termed WDLS [2], whereas those located in the extremities and superficial sites are classified as ALT [1].

It has been difficult to differentiate between ALT/WDLS and lipoma preoperatively because their magnetic resonance imaging (MRI) findings are similar [1], and small biopsy samples are insufficient for 
histopathological diagnosis [7]. The selection of surgical resection margins in these tumors remains controversial. ALT and WDLS are classified as intermediate (locally aggressive) and malignant tumors, respectively. They rarely metastasize, but a risk of local recurrence of them is higher than lipoma [7]. Additionally, as they may undergo dedifferentiation and become malignant tumors [8], some patients need wider resection margins while still avoiding dysfunction. Therefore, the preoperative differential diagnosis between ALT/WDLS and lipoma is critical for the determination of the resection margin.

Although there have been some reports regarding the usefulness and high accuracy of the assessment, such as fluorescence in situ hybridization (FISH) for murine double-minute 2 (MDM2) and cyclindependent kinase 4 (CDK4) for the diagnosis of ALT/WDLS [9-16], these technologies requires special equipment and reagents, so not all medical institutions can perform this examination [7]. The assessment with MRI and hematoxylin and eosin (HE) staining remains the standard method of diagnosis for adipocytic tumors, so it is crucial to determine the diagnosis by a comprehensive assessment of clinical, radiological, and histopathological examinations. Here, we evaluated the diagnostic accuracy of various findings in some examinations and aimed to develop a combined scoring system based on the accuracy of each finding for the preoperative differential diagnosis between ALT/WDLS and lipoma.

\section{Materials And Methods}

\section{Study design and patients}

This retrospective study included patients diagnosed with ALT/WDLS or lipoma from 2005 to 2019 collected from the database at Kanazawa University Hospital. A total of 297 patient charts were extracted, and 145 patients who underwent surgical resection after evaluation by MRI and needle biopsy were included in this study. The diagnoses were established by pathologists based on the resected specimens. Spindle cell lipoma and pleomorphic lipoma which have relatively typical pathological findings, and tumor recurrences were excluded from this study. This study was approved by the ethics committee of Kanazawa University Hospital, and the individual consent was obtained by the opt-out method.

\section{Data analyses}

We collected the patients' clinical characteristics, including age, sex, symptoms (pain and numbness), and tumor site and depth from the medical records. The tumor depth was defined as a superficial or deep site with the fascia as the boundary by MRI. Based on the previous studies regarding MRI findings used to distinguish ALT/WDLS and lipoma, radiologists evaluated three criteria: thick septa (>2mm), enhancement of septa or nodular lesions, and neurovascular involvement in the tumor $[1,17,18]$ (Fig. 1). Furthermore, based on histological findings by HE staining from needle biopsies specimens, the pathologists evaluated characteristics such as nuclear atypia and enlargement, proliferation of fibrous septa, differences in the size of adipocytes, and lipoblasts [2, 8, 19] (Fig. 2). Lipoblasts were defined as the cells having hyperchromatic indented or sharply scalloped nuclei and lipid-rich mono- or 
multivacuolated cytoplasm [8, 20]. In most patients diagnosed after 2013, the needle biopsy specimens were also used to evaluate FISH for MDM2 and CDK4.

\section{Statistical analyses}

These characteristics and findings were compared between the ALT/WDLS and lipoma groups by Student's t-test or the chi-square test. A p-value of less than 0.05 was considered statistically significant. Univariate logistic regression analysis was performed to determine the predictive factors for the diagnosis of ALT/WDLS and variables with $p<0.05$ from univariate analysis were used for multivariate logistic regression analysis. A combined scoring system for the diagnosis of ALT/WDLS was developed according to the predictive factors that showed statistical significance by multivariate analyses. The accuracy of the diagnoses of the scoring system was evaluated using a receiver operating characteristic (ROC) curve. All data analyses were performed using EZR [21].

\section{Results}

The 145 participants comprised 77 men and 68 women, with a mean age of $58.8 \pm 14.7$ years (range 1887 years). Based on the diagnoses established by the pathologists, the numbers of patients in the ALT/WDLS and lipoma groups were 89 and 56, respectively (Table 1). Clinical findings showed that the mean age of patients in the ALT/WDLS group was significantly higher than that in the lipoma group $(64.0 \pm 13.3$ vs. $55.7 \pm 14.6, p<0.001)$. All tumors in the ALT/WDLS group were located deep $(100 \%$ vs. $67.4 \%, p<0.0001)$ and significantly larger than those in the lipoma group $(17.2 \pm 6.7 \mathrm{~cm}$ vs. $9.7 \pm 4.2 \mathrm{~cm}, p$ $<0.0001$ ) (Table 1). On MRI, every criterion showed a significant difference between the ALT/WDLS and lipoma groups (thick septa $(>2 \mathrm{~mm}) ; p<0.0001$; enhancement; $p<0.0001$; and neurovascular involvement, $p=0.0225$ ). The histological findings by HE staining, such as nuclear atypia and enlargement, difference in size of adipocytes and lipoblasts, and FISH examination for MDM2 and/or CDK4 showed a significant differences between them $(p<0.0001)$. Only the difference in the size of adipocytes has a non-significant p-value of 0.0162 (Table 1). 
Table 1

Characteristics of patients, MRI, and histopathological findings

\begin{tabular}{|c|c|c|c|}
\hline & $\begin{array}{l}\text { ALT/WDLS }(N= \\
56)\end{array}$ & Lipoma $(\mathrm{N}=89)$ & p-value \\
\hline Clinical findings & $\begin{array}{l}64.0 \pm 13.29(30- \\
87)\end{array}$ & $\begin{array}{l}55.7 \pm 14.60(18- \\
83)\end{array}$ & \multirow{7}{*}{$\begin{array}{l}<0.001 \\
0.547 \\
0.150\end{array}$} \\
\hline Age & \multirow{2}{*}{$\begin{array}{l}32(57.1 \%) \\
24(42.9 \%)\end{array}$} & \multirow{2}{*}{$45(50.6 \%)$} & \\
\hline Median (range) & & & \\
\hline Sex: n (\%) & \multirow{4}{*}{$6(10.7 \%)$} & \multirow{4}{*}{$4(4.5 \%)$} & \\
\hline Male & & & \\
\hline Female & & & \\
\hline Symptoms: n (\%) & & & \\
\hline Tumor site: n (\%) & $5(8.9 \%)$ & $29(32.6 \%)$ & \multirow{4}{*}{$\dot{0} 0001$} \\
\hline Upper limb & $39(69.7 \%)$ & $24(27.0 \%)$ & \\
\hline Lower limb & \multirow[t]{2}{*}{$12(21.4 \%)^{a}$} & \multirow[t]{2}{*}{$36(40.4 \%)^{b}$} & \\
\hline Other & & & \\
\hline Tumor depth: n (\%) & $0(0 \%)$ & $29(32.6 \%)$ & \multirow{3}{*}{$\dot{0} 0001$} \\
\hline Superficial & \multirow[t]{2}{*}{$56(100 \%)$} & \multirow[t]{2}{*}{$60(67.4 \%)$} & \\
\hline Deep & & & \\
\hline Tumor size (cm) & \multirow[t]{2}{*}{$17.2 \pm 6.7$} & \multirow[t]{2}{*}{$9.7 \pm 4.2$} & \multirow{2}{*}{$<.0001$} \\
\hline Median & & & \\
\hline MRI findings: n (\%) & $52(92.9 \%)$ & $52(58.4 \%)$ & \multirow{2}{*}{$\begin{array}{l}< \\
0.0001\end{array}$} \\
\hline Thick septa (> 2mm) & $20(100 \%)$ & $4(20.0 \%)$ & \\
\hline $\begin{array}{l}\text { Enhancement (septa and/or nodular lesions) } \\
(\mathrm{n}=20)\end{array}$ & \multirow[t]{2}{*}{$5(8.9 \%)$} & \multirow[t]{2}{*}{$1(1.1 \%)$} & 0.0001 \\
\hline Neurovascular involvement & & & 0.023 \\
\hline
\end{tabular}

\footnotetext{
a neck $(n=4)$, chest $(n=2)$, retroperitoneum $(n=2)$, and back $(n=2)$

${ }^{b}$ neck $(n=15)$, chest $(n=13)$, back $(n=6)$, and abdomen $(n=2)$

Abbreviations: $M R /$ magnetic resonance imaging, $A L T$ atypical lipomatous tumor, WDLS welldifferentiated liposarcoma, FISH Fluorescence in Situ Hybridization, MDM2 murine double-minute 2, CDK4 cyclin-dependent kinase 4
} 


\begin{tabular}{|c|c|c|c|}
\hline & $\begin{array}{l}\text { ALT/WDLS ( }= \\
56)\end{array}$ & Lipoma ( $=89)$ & p-value \\
\hline Histopathological findings: n (\%) & $10(17.9 \%)$ & $0(0 \%)$ & \multirow{2}{*}{$\begin{array}{l}< \\
0.0001\end{array}$} \\
\hline Nuclear atypia & $26(46.4 \%)$ & $8(9.0 \%)$ & \\
\hline Nuclear enlargement & $26(46.4 \%)$ & $24(27.0 \%)$ & 0.0001 \\
\hline Difference in size of adipocytes & $2(3.6 \%)$ & $3(3.4 \%)$ & 0.016 \\
\hline Proliferation of fibrous septa & $11(19.6 \%)$ & $1(1.1 \%)$ & 0.949 \\
\hline Lipoblasts & & & $<0.0001$ \\
\hline \multirow{2}{*}{$\begin{array}{l}\text { FISH: positive number / } \mathrm{n}=\text { total number }(\%) \\
\text { MDM2 }\end{array}$} & $\begin{array}{l}37 / n=46 \\
(80.4 \%)\end{array}$ & $0 / n=73(0 \%)$ & \multirow{2}{*}{$\begin{array}{l}< \\
0.0001 \\
< \\
0.0001\end{array}$} \\
\hline & $\begin{array}{l}18 / n=33 \\
(54.5 \%)\end{array}$ & $0 / n=49(0 \%)$ & \\
\hline \multicolumn{4}{|c|}{ a neck $(n=4)$, chest $(n=2)$, retroperitoneum $(n=2)$, and back $(n=2)$} \\
\hline \multicolumn{4}{|c|}{ b neck $(n=15)$, chest $(n=13)$, back $(n=6)$, and abdomen $(n=2)$} \\
\hline \multicolumn{4}{|c|}{$\begin{array}{l}\text { Abbreviations: } M R / \text { magnetic resonance imaging, } A L T \text { atypical lipomatous tumor, WDLS well- } \\
\text { differentiated liposarcoma, FISH Fluorescence in Situ Hybridization, MDM2 murine double-minute 2, } \\
\text { CDK4 cyclin-dependent kinase } 4\end{array}$} \\
\hline
\end{tabular}

The tumor size was analyzed using the ROC curve, with the cutoff value was set to $11 \mathrm{~cm}$. In previous studies, cutoff values of tumor size for differential diagnosis between lipoma and ALT/WDLS were 10$13 \mathrm{~cm}[1,22]$, and our result was consistent with those studies. Univariate analyses revealed that age, tumor location, depth and size, thick septa (>2 $\mathrm{mm}$ ), enhancement of septa or nodular lesions, histological findings excluding the proliferation of fibrous septa, and FISH examination for MDM2 and/or CDK4 were significantly different (Table 2). Among these factors, the odds ratio of tumor depth, nuclear atypia, and FISH examination were extremely higher than other factors. Particularly, nuclear atypia and FISH examination were not positive in the lipoma group in this study, and the specificity of these parameters for the diagnosis of ALT/WDLS was $100 \%$. If nuclear atypia is found in the specimens of the adipocytic tumor, the diagnosis of lipoma is excluded. MDM2 and CDK4 gene amplification by FISH examination is considered the gold standard for the differential diagnosis between ALT/WDLS and lipoma [15], and these gene amplifications are not observed in lipoma. From these results, nuclear atypia and FISH examination, which are used for the definitive diagnosis of ALT/WDLS, were excluded in this study. We aimed to develop a scoring system in which these factors were negative. 
Table 2

Univariate and multivariate logistic regression analysis of predictive factors for ALT/WDLS

\begin{tabular}{|c|c|c|c|c|}
\hline & \multicolumn{2}{|l|}{ Univariate analysis } & \multicolumn{2}{|c|}{ Multivariate analysis } \\
\hline & $\begin{array}{l}\text { Odds ratio (95\% } \\
\mathrm{Cl})\end{array}$ & p-value & $\begin{array}{l}\text { Odds ratio }(95 \% \\
\mathrm{Cl})\end{array}$ & p-value \\
\hline Clinical findings & $4.26(1.87-9.74)$ & $<0.001$ & $4.16(0.93-18.60)$ & 0.062 \\
\hline Age & $1.30(0.67-2.56)$ & 0.440 & & \\
\hline Sex & $2.55(0.69-9.47)$ & 0.162 & & \\
\hline \multicolumn{5}{|l|}{ Symptoms } \\
\hline Tumor site & $6.21(2.97-13.00)$ & \multirow{2}{*}{$\dot{0.0001}$} & $5.51(1.51-20.10)$ & 0.001 \\
\hline Lower limb vs Upper limb or Other & \multirow[t]{2}{*}{$>1000$ (0.00-Inf) } & & $>1000$ (0.00-Inf) & $<$ \\
\hline Depth & & \multicolumn{2}{|l|}{$\begin{array}{l}< \\
0.0001\end{array}$} & \\
\hline Tumor size (> 11cm) & $\begin{array}{l}12.40(5.20- \\
29.60)\end{array}$ & $<.0001$ & $4.83(1.37-17.00)$ & 0.014 \\
\hline \multirow{4}{*}{$\begin{array}{l}\text { MRI findings } \\
\text { Thick septa (>2mm) } \\
\text { Enhancement (septa or nodular } \\
\text { lesion) } \\
\text { Neurovascular involvement }\end{array}$} & $9.25(3.08-$ & \multirow{2}{*}{$<.0001$} & $5.56(1.09-28.30)$ & 0.039 \\
\hline & & & \multirow{3}{*}{$\begin{array}{l}15.10(2.91- \\
77.90)\end{array}$} & \multirow[t]{3}{*}{0.001} \\
\hline & 76.10) & 0.0001 & & \\
\hline & $\begin{array}{l}8.63(0.98- \\
75.90)\end{array}$ & 0.052 & & \\
\hline Histopathological findings & $>1000$ (0.00-Inf) & \multirow{2}{*}{$<.0001$} & $1.69(0.40-7.17)$ & 0.480 \\
\hline Nuclear atypia & $8.77(3.58-$ & & $0.91(0.26-3.20)$ & 0.888 \\
\hline Nuclear enlargement & & $\begin{array}{l}<.0001 \\
0 .\end{array}$ & \multirow{4}{*}{$\begin{array}{l}17.60(1.08- \\
286.00)\end{array}$} & \multirow[t]{4}{*}{0.043} \\
\hline Difference in size of adipocytes & $2.00(1.10-4.14)$ & 0.018 & & \\
\hline Proliferation of fibrous septa & \multirow{2}{*}{$\begin{array}{l}21.5(2.69- \\
172.00)\end{array}$} & \multirow{2}{*}{$\begin{array}{l}0.949 \\
0.004\end{array}$} & & \\
\hline Lipoblasts & & & & \\
\hline $\mathrm{FISH}$ & \multirow[t]{2}{*}{$>1000$ (0.00-Inf) } & \multirow{2}{*}{$\begin{array}{l}<.0001 \\
0.01\end{array}$} & & \\
\hline MDM2 and/or CDK4 & & & & \\
\hline $\begin{array}{l}\text { Abbreviations: } M R / \text { magnetic reson } \\
\text { lipomatous tumor, } W D L S \text { well-diffe } \\
M D M 2 \text { murine double-minute } 2, C D\end{array}$ & $\begin{array}{l}\text { e imaging, } \mathrm{Cl} \text { conf } \\
\text { iated liposarcoma } \\
\text { cyclin-dependent } k\end{array}$ & $\begin{array}{l}\text { nce interv } \\
\text { SH Fluore } \\
\text { se } 4\end{array}$ & $\begin{array}{l}\text { I, Inf infinity, } A L T \text { aty } \\
\text { cence in Situ Hybridi }\end{array}$ & $\begin{array}{l}\text { cal } \\
\text { tion, }\end{array}$ \\
\hline
\end{tabular}

Subsequently, multivariate analysis incorporating the variables with $p<0.05$ in the univariate analysis was performed. In the analysis, tumor location (lower extremity), depth (deep site), and size $(>11 \mathrm{~cm})$, 
thick septa $(>2 \mathrm{~mm})$, enhancement of septa or nodular lesions, and lipoblasts were significantly different, and they had a high accuracy in the diagnosis of ALT/WDLS (Table 2).

Based on these results, a combined scoring system for the preoperative differential diagnosis of ALT/WDLS was developed according to the predictive factors (Table 3). With reference to the odds ratio, the points of this score were set as follows: $<5,1$ point; $5-10,2$ points; and $>10,3$ points. The odds ratio of the tumor depth was extremely high, so the point was set to 5 points. The total points ranged from 0 to 16 , and the mean number of points of the ALT/WDLS group was significantly higher than that of the lipoma group (mean points of ALT/WDLS and lipoma groups were 11.9 vs. 5.6, $p<0.0001$ ). The cutoff value was 9 points based on the ROC curve analysis. The increased points suggested that the possibility of ALT/WDLS was higher, with a sensitivity of $87.6 \%$ and a specificity of $91.1 \%$ (Fig. 3). The analysis of the ROC curve also showed an area under the curve (AUC) of 0.945, which indicated that the accuracy of this combined scoring system for the differential diagnosis of ALT/WDLS was very high. If needle biopsy could not be performed, the total points were ranged from 0 to 13, excluding lipoblast, and the ROC curve analysis showed the cutoff value was 10 points and AUC was 0.943 with a sensitivity of $94.4 \%$ and a specificity of $80.4 \%$.

Table 3. A combined scoring system for the preoperative differential diagnosing of ALT/WDLS The total points ranged from 0 to 16, and the cutoff value was 9 points.

\begin{tabular}{|c|c|c|}
\hline Predictive factors & Odds ratio & Points \\
\hline $\begin{array}{l}\text { Tumor size (MRI) } \\
<11 \mathrm{~cm} \\
\geq 11 \mathrm{~cm}\end{array}$ & 4.83 & $\begin{array}{l}0 \\
1\end{array}$ \\
\hline $\begin{array}{l}\text { Tumor location (MRI) } \\
\text { Upper limb or Other* } \\
\text { Lower limb }\end{array}$ & 5.51 & $\begin{array}{l}0 \\
2\end{array}$ \\
\hline $\begin{array}{l}\text { Thick septa }(>2 \mathrm{~mm})(\mathrm{MRI}) \\
\text { No } \\
\text { Yes }\end{array}$ & 5.56 & $\begin{array}{l}0 \\
2\end{array}$ \\
\hline $\begin{array}{l}\text { Enhancement of septa or nodular lesion (Enhanced MRI) } \\
\text { No } \\
\text { Yes }\end{array}$ & 15.10 & $\begin{array}{l}0 \\
3\end{array}$ \\
\hline $\begin{array}{l}\text { Lipoblast (HE staining) } \\
\text { Negative } \\
\text { Positive }\end{array}$ & 17.60 & $\begin{array}{l}0 \\
3\end{array}$ \\
\hline $\begin{array}{l}\text { Depth (MRI) } \\
\text { Superficial } \\
\text { Deeper than fascia }\end{array}$ & $>1000$ & $\begin{array}{l}0 \\
5\end{array}$ \\
\hline Cuttoff value & & $9 / 16$ points \\
\hline
\end{tabular}

*: neck, chest, abdomen, back, and retroperitoneum 
Abbreviations: $M R I$ magnetic resonance imaging, $H E$ hematoxylin and eosin, $A L T$ atypical lipomatous tumor, WDLS well-differentiated liposarcoma

\section{Discussion}

Orthopedic surgeons sometimes treat soft tissue tumors, and they sometimes encounter adipocytic tumors that must be diagnosed and treated. A lipoma is a benign tumor that can usually be treated conservatively $[1,7]$. Even if the tumor size is large, surgical treatment is not necessary as long as it remains asymptomatic. On the other hand, ALT/WDLS is the most common subtype of liposarcoma, and the treatment for these tumors is controversial [1] because it is reported that $1-4 \%$ of them undergo dedifferentiation [23-25] and may become malignant tumors [2, 8, 23, 26]. If preoperative differential diagnosis between ALT/WDLS and lipoma is easy in ALT/WDLS patients, surgical resection is recommended before dedifferentiation [1]. The decision of the resection margin of ALT/WDLS is difficult because the local recurrence rate is high [7]. It has been reported that there was no significant difference in the recurrence rate between the wide and marginal resection in ALT [27], and "conservative" surgery, aiming to preserve major vessels or nerves, may be recommended for deep-seated ALT/WDLS [28-31]. However, insufficient resection may increase the risk of recurrence and dedifferentiation and resulting in the need for more surgery to perform additional tissue resection. We have reported studies on treatment strategies for ALT/WDLS [29], and based on the results, if the border between the normal tissue and ALT/WDLS of the limb is ill-defined, sub-extensive resection is performed including the surrounding soft tissue, such as muscle tissue. If the border is well-defined between such a tumor and neurovascular bundles, marginal resection and sub-extensive resection are performed. On the other hand, in recent years, Vos et al. reported that observation could be a reasonable option for selected patients with extremity WDLS [32]. For these reasons, it is crucial to make an accurate differential diagnosis between ALT/WDLS and lipoma for the preoperative plan of the appropriate resection margin and the appropriate selection of patients who can be observed.

For the differential diagnosis, previous studies reported critical factors such as clinical findings (older age $[13,33,34]$, tumor size [>10cm] [1, 12,33,35], tumor site [lower extremity] [12, 33, 35], and deep-seated location [1]) and MRI findings (thick septa [> 2mm] [17, 18], fat content less than 75\% [34], and contrast enhancement [1]).

In this study, a multivariate analysis was performed to evaluate the predictive factors for ALT/WDLS. There was a significant difference in tumor site (lower extremity), depth, size $(>11 \mathrm{~cm})$, thick septa, and enhancement of septa or nodular lesions. All of these were consistent with the previously mentioned factors, demonstrating high accuracy for the differential diagnosis of ALT/WDLS. MRI findings have high sensitivity but low specificity for the diagnosis of ALT/WDLS, and it has been reported that it may be difficult to decide the diagnosis of them by only MRI findings [7, 33]. On the other hand, Brisson et al. reported that histopathological examination could be used to distinguish ALT/WDLS from lipomas [33], so for adipocytic tumors, needle biopsies have been generally performed preoperatively to obtain the diagnosis [2]. 
Histopathological findings in the multivariate analysis showed that lipoblasts were statistically significant for the diagnosis of ALT/WDLS. Traditionally, lipoblasts have been emphasized as a histopathological finding in the diagnosis of liposarcoma [36]. However, lipoblasts are also observed in benign lipogenic tumors such as spindle cell/pleomorphic lipoma. Furthermore, it has been reported that they are not always observed in ALT/WDLS [37]. Although the diagnostic accuracy of lipoblasts in the diagnosis of ALT/WDLS has not been clarified, our study revealed that their sensitivity was low (19.6\%), but the specificity was very high (98.9\%). In the combined scoring system, it is implied that lipoblasts are crucial findings for the diagnosis of ALT/WDLS.

In this study, nuclear atypia had a significant difference in the differential diagnosis of ALT/WDLS by univariate analysis. ALT/WDLS features a mature adipocytic tumor showing atypical hyperchromatic nuclei [2], which is consistent with our results. No nuclear atypia is found in lipoma, which is a benign tumor, so this characteristic is used for the rule out of the diagnosis of these tumors. The specificity of nuclear atypia was $100 \%$ in the diagnosis of ALT/WDLS in this study. However, needle biopsies may not provide enough sample for the identification of unequivocal atypical cells [7, 38], and the atypical stromal cells sometimes scatter throughout the lesion; therefore, in some cases, the difference between ALT/WDLS and lipoma may be subtle challenging the differential diagnosis process [38]. In addition, FISH examination for MDM2 and CDK4 gene amplification has provided the most accuracy for the diagnosis of ALT/WDLS $[9-13,15,16]$, and it is considered the gold standard for the differential diagnosis between ALT/WDLS and lipoma [15]. In this study, as well as previous studies, MDM2 and/or CDK4 amplification by FISH examination showed a significant difference as a predictive factor for ALT/WDLS. Furthermore, the specificity was 100\%. MDM2 gene amplification in FISH examination has high sensitivity and specificity [15], and this finding has been used for the definitive diagnosis of ALT/WDLS. However, similar to nuclear atypia, an insufficient sample and the selection of an inappropriate needle biopsy site might complicate the accurate exclusion of a diagnosis of ALT/WDLS due to the absence of MDM2 gene amplification. Furthermore, HE staining in the histopathological examination can be generally performed in many institutions, but FISH examination requires special equipment and reagents, which not all institutions are equipped to perform [7]. For these reasons, this study excluded the nuclear atypia and FISH examination used for the definitive diagnosis of ALT/WDLS and aimed to develop a scoring system for the differential diagnosis of ALT/WDLS in case where these factors were negative.

Therefore, in adipocytic tumors, the differential diagnosis should be evaluated based on a comprehensive assessment of clinical, radiological, and histopathological examinations. Although a few scoring systems for the differential diagnosis of ALT/WDLS based on radiological findings have been reported [1, 7], there is no diagnostic scoring system that includes histopathological findings. Here, we aimed to develop a new combined scoring system, including clinical, radiological, and histopathological findings, to easily perform the preoperative differential diagnosis between ALT/WDLS and lipoma.

A combined scoring system with 6 predictive factors for ALT/WDLS that had a significant difference in multivariate analysis was developed. The total points of this scoring system were 16 points, and the ROC 
curve analysis showed a sensitivity of $87.6 \%$, a specificity of $91.1 \%$, and an AUC of 0.945 in the diagnosis of ALT/WDLS. The cutoff value was 9 points, and the possibility of ALT/WDLS increased as the total score increased.

In the previous studies of similar scoring systems, Nagano et al. reported a sensitivity of $100 \%$ and specificity of $77 \%$ [1], and Cheng et al. reported a sensitivity of $90 \%$ and specificity of $92.5 \%$ [7]. The diagnostic accuracy for ALT/WDLS of this scoring system is almost the same as in these studies. However, Nagano et al. study examined 48 lipomas and 12 ALTs, without including WDLS, and the case number was lower compared with our study [1]. Although this study investigated adipocytic tumors in all locations, Cheng et al. investigated only deep-seated adipocytic tumors [7]. These limitations may have affected the difference in accuracy between our scoring system and those studies.

In this study, our combined scoring system could not improve FISH for MDM2 in ALT/WDLS. Although high sensitivity and specificity of FISH for MDM2 in ALT/WDLS have been reported in previous studies, FISH for MDM2 and has been performed in limited institutions. This scoring system may help to differentiate between lipoma and ALT/WDLS in cases without the assessment of MDM2 amplification.

The histopathological findings included in this scoring system are based on the specimens of preoperative needle biopsy. Therefore, this is a limitation of this study because the evaluation of these specimens may be affected by the biopsy site and the amount of sample, and not all medical institutions can perform a needle biopsy. If needle biopsy cannot be performed, our scoring system can use 5 predictive factors except lipoblast and the cutoff value was 10 points in total 13 points. Since the cutoff value of this scoring system including lipoblast was 9 points, a needle biopsy may be recommended in the case of 6-8 points in the scoring system except for lipoblast. Furthermore, if a preoperative needle biopsy can be performed with an accurate procedure, the diagnostic accuracy for ALT/WDLS of this scoring system may be higher, which makes it a very useful diagnostic tool for the preoperative differential diagnosis of adipocytic tumors.

\section{Conclusions}

We developed a new combined scoring system based on a comprehensive assessment of clinical, radiological, and histopathological examinations for the preoperative differential diagnosis between ALT/WDLS and lipoma. Multivariate analysis revealed the predictive factors for ALT/WDLS such as tumor site (lower extremity), location (deep site), and size $(>11 \mathrm{~cm})$, thick septa $(>2 \mathrm{~mm})$, enhancement of septa or nodular lesions, and lipoblasts. This scoring system based on these 6 predictive factors had high diagnostic accuracy for differential diagnosis of ALT/WDLS and it is a useful preoperative diagnostic tool if nuclear atypia in the histopathological examination was negative or the FISH examination for MDM2 and CDK4 cannot perform.

\section{Abbreviations}


ALT: atypical lipomatous tumor; WDLS: well-differentiated liposarcoma; MRI: magnetic resonance imaging; HE: hematoxylin and eosin; FISH: fluorescence in situ hybridization; MDM2: murine doubleminute 2; CDK4: cyclin-dependent kinase 4; HE: hematoxylin and eosin; ROC: receiver operating characteristic; AUC: area under the curve

\section{Declarations}

\section{Ethics approval and consent to participate}

Research of this study involving human material and data have been performed in accordance with the Declaration of Helsinki, and the Institutional Review Board (IRB) at the Kanazawa University Hospital approved the study. Each author certifies that all investigations were conducted in conformity with the ethical principles. Written informed consent was obtained from all patients included in the study by the opt-out method.

\section{Consent for publication}

Not applicable.

\section{Availability of data and materials}

All data generated or analyzed during this study are included in this published article and there are no unpublished data

\section{Competing interests}

The authors declare that they have no competing interests.

\section{Funding}

Not applicable.

\section{Authors' contributions}

All authors were involved in the preparation of this study. YA and SM collected the data and performed the operation, and YA wrote the manuscript. NY, KH, AT, KI, HY, YA, and SM performed the operation and designed the study. TN and HI performed a histopathological evaluation of the specimens. HT revised the manuscript. All authors read and approved the final manuscript.

Acknowledgments: We would like to thank Editage (www.editage.jp) for English language editing.

\section{References}


1. Nagano S, Yokouchi M, Setoguchi T, Ishidou Y, Sasaki H, Shimada H, et al. Differentiation of lipoma and atypical lipomatous tumor by a scoring system: implication of increased vascularity on pathogenesis of liposarcoma. BMC Musculoskelet Disord. 2015;16:36.

2. The World Health Organization classification of Tumor Editorial Board. WHO Classification of Tumors Soft Tissue and Bone Tumors. 5th ed. Lyon: IARC Press; 2020.

3. Dalal KM, Antonescu CR, Singer S. Diagnosis and management of lipomatous tumors. J Surg Oncol. 2008;97:298-313.

4. Coindre JM. New WHO classification of tumors of soft tissue and bone. Ann Pathol 2012;32 Suppl 5:115-116.

5. Wang J, Zhu XZ. Introduction of WHO classification of tumours of soft tissue, the fourth edition. Zhonghua Bing Li Xue Za Zhi. 2013;42:363-365.

6. Conyers R, Young S, Thomas DM. Liposarcoma: Molecular Genetics and Therapeutics. Sarcoma. 2011; doi:10.1155/2011/483154.

7. Cheng Y, Ko AT, Huang JH, Lee BC, Yang RS, Liang CW, et al. Developing a clinical scoring system to differentiated deep-seated atypical lipomatous tumor from lipoma of soft tissue. Asian J Surg. 2019;42:832-838.

8. Laurino L, Furlanetto A, Orvieto E, Dei Tos AP. Well-differentiated liposarcoma (atypical lipomatous tumors). Semin Diagn Pathol. 2001;18:258-262.

9. Binh MB, Sastre-Garau X, Guillou L, de Oinieux G, Terrier P, Lagacé R, et al. MDM2 and CDK4 immunostainings are useful adjuncts in diagnosing well-differentiated and dedifferentiated liposarcoma subtypes: a comparative analysis of 559 soft tissue neoplasms with genetic data. Am J Surg Pathol. 2005;29:1340-1347.

10. Kimura H, Dobashi Y, Nojima T, Nakamura H, Yamamoto N, Tsuchiya H, et al. Utility of fluorescence in situ hybridization to detect MDM2 amplification in liposarcomas and their morphological mimics. Int J Clin Exp Pathol. 2013;6:1306-1316.

11. Clay MR, Martinez AP, Weiss SW, Edgar MA. MDM2 Amplification in Problematic Lipomatous Tumors: Analysis of FISH Testing Criteria. Am J Surg Pathol. 2015;39:1433-1439.

12. Fisher SB, Baxter KJ, Staley CA 3rd, Fisher KE, Monson DK, Murray DR, et al. The General surgeon's quandary: atypical lipomatous tumor vs lipoma, who needs a surgical oncologist? J Am Coll Surg. 2013;217:881-888.

13. Weaver J, Rao P, Goldblum JR, Joyce MJ, Turner SL, Lazar AJF, et al. Can MDM2 analytic tests performed on core needle biopsy be relied upon to diagnose well-differentiated liposarcoma? Mod Pathol. 2010;23:1301-1306.

14. Kulkarni AS, Wojcik JB, Chougule A, Arora K, Chittampalli Y, Kurzawa P, et al. MDM2 RNA In Situ Hybridization for the Diagnosis of Atypical Lipomatous Tumor: A Study Evaluating DNA, RNA, and Protein Expression. Am J Surg Pathol. 2019;43:446-454.

15. Kashima T, Halai D, Ye H, Hing SN, Delaney D, Pollock R, et al. Sensitivity of MDM2 amplification and unexpected multiple faint alphoid 12 (alpha 12 satellite sequences) signals in atypical lipomatous 
tumor. Mod Pathol. 2012;25:1384-1396.

16. Dei Tos AP, Doglioni C, Piccinin S, Sciot R, Furlanetto A, Boiocchi M, et al. Coordinated expression and amplification of the MDM2, CDK4, and HMGI-C genes in atypical lipomatous tumours. J Pathol. 2000;190:531-536.

17. Arkun R, Memis A, Akalin T, Ustun EE, Sabah D, Kandiloglu G. Liposarcoma of soft tissue: MRI findings with pathologic correlation. Skeletal Radiol. 1997;26:167-172.

18. Ohguri T, Aoki T, Hisaoka M, Watanabe H, Nakamura K, Hashimoto H, et al. Differential diagnosis of benign peripheral lipoma from well-differentiated liposarcoma on MR imaging: is comparison of margins and internal characteristics useful? AJR Am J Roentgenol. 2003;180:1689-1694.

19. Lee ATJ, Thway K, Huang PH, Jones RL. Clinical and Molecular Spectrum of Liposarcoma. J Clin Oncol. 2018;36:151-159.

20. Goldblum JR, Folpe AL, Weiss SW. Enzinger \& Weiss's Soft Tissue Tumors. 6th ed. Philadelphia: Elsevier; 2013. p. 484-523.

21. Kanda Y. Investigation of the freely available easy-to-use software 'EZR' for medical statistics. Bone Marrow Transplant. 2013;48:452-458.

22. Knebel C, Neumann J, Schwaiger BJ, Karampinos DC, Pfeiffer D, Specht K, et al. Differentiating atypical lipomatous tumors from lipomas with magnetic resonance imaging: a comparison with MDM2 gene amplification status. BMC Cancer. 2019;19:309.

23. Sommerville SM, Patton JT, Luscombe JC, Mangham DC, Grimer RJ. Clinical outcomes of deep atypical lipomas (well-differentiated lipoma-like liposarcomas) of the extremities. ANZ J Surg. 2005;75:803-806.

24. Bassett MD, Schuetze SM, Disteche C, Norwood TH, Swisshelm K, Chen X, et al. Deep-seated, well differentiated lipomatous tumors of the chest wall and extremities: the role of cytogenetics in classification and prognostication. Cancer, 2005;103:409-416.

25. Kooby DA, Antonescu CR, Brennan MF, Singer S. Atypical lipomatous tumor/well-differentiated liposarcoma of the extremity and trunk wall: importance of histological subtype with treatment recommendations. Ann Surg Oncol. 2004;11:78-84.

26. Lucas DR, Nascimento AG, Sanjay BK, Rock MG. Well-differentiated liposarcoma. The Mayo Clinic experience with 58 cases. Am J Clin Pathol. 1994;102:677-683.

27. Mussi CE, Daolio P, Cimino M, Giardina F, De Sanctis R, Morenghi E, et al. Atypical lipomatous tumors: should they be treated like other sarcoma or not? Surgical consideration from a biinstitutional experience. Ann Surg Oncol. 2014;21:4090-4097.

28. Chang DH, Ma H, Liao WC, Huang MH, Wu PS. Atypical lipomatous tumors of the extremities and trunk wall-the first case series of Chinese population with 45 cases. Ann Plast Surg 2016;76 Suppl $1: 8-12$.

29. Yamamoto N, Hayashi K, Tanzawa Y, Kimura H, Takeuchi A, Igarashi K, et al. Treatment strategies for well-differentiated liposarcomas and therapeutic outcomes. Anticancer Res. 2012;32:1821-1825. 
30. Gronchi A, Lo Vullo S, Colombo C, Collini P, Stacchiotti S, Mariani L, et al. Extremity soft tissue sarcoma in a series of patients treated at a single institution: local control directly impacts survival. Ann Surg. 2010;251:506-511.

31. Kubo T, Sugita T, Shimose S, Arihiro K, Ochi M. Conservative surgery for well-differentiated liposarcomas of the extremities adjacent to major neurovascular structures. Surg Oncol. 2006;15:167-171.

32. Vos M, Grünhagen DJ, Koseła-Paterczyk H, Rutkowski P, Sleijfer S, Verhoef C. Natural history of welldifferentiated liposarcoma of the extremity compared to patients treated with surgery. Surg Oncol. 2019;29:84-89.

33. Brisson M, Kashima T, Delaney D, Tirabosco R, Clarke A, Cro S, et al. MRI characteristics of lipoma and atypical lipomatous tumor/well-differentiated liposarcoma: retrospective comparison with histology and MDM2 gene amplification. Skeletal Radiol. 2013;42:635-647.

34. Kransdorf MJ, Bancroft LW, Peterson JJ, Murphey MD, Foster WC, Temple HT. Imaging of fatty tumors: distinction of lipoma and well-differentiated liposarcoma. Radiology. 2002;224:99-104.

35. Bird JE, Morse LJ, Feng L, Wang WL, Lin PP, Moon BS, et al. Non-radiographic risk factors differentiating atypical lipomatous tumors from lipomas. Front Oncol. 2016;6:197.

36. Hisaoka M. Lipoblast: morphological features and diagnostic value. J UOEH. 2014;36:115-121.

37. Dei Tos AP. Liposarcomas: diagnostic pitfalls and new insights. Histopathology. 2014;64:38-52.

38. Coindre JM, Pedeutour F, Aurias A. Well-differentiated and dedifferentiated liposarcomas. Virchows Arch. 2010;456:167-179.

\section{Figures}
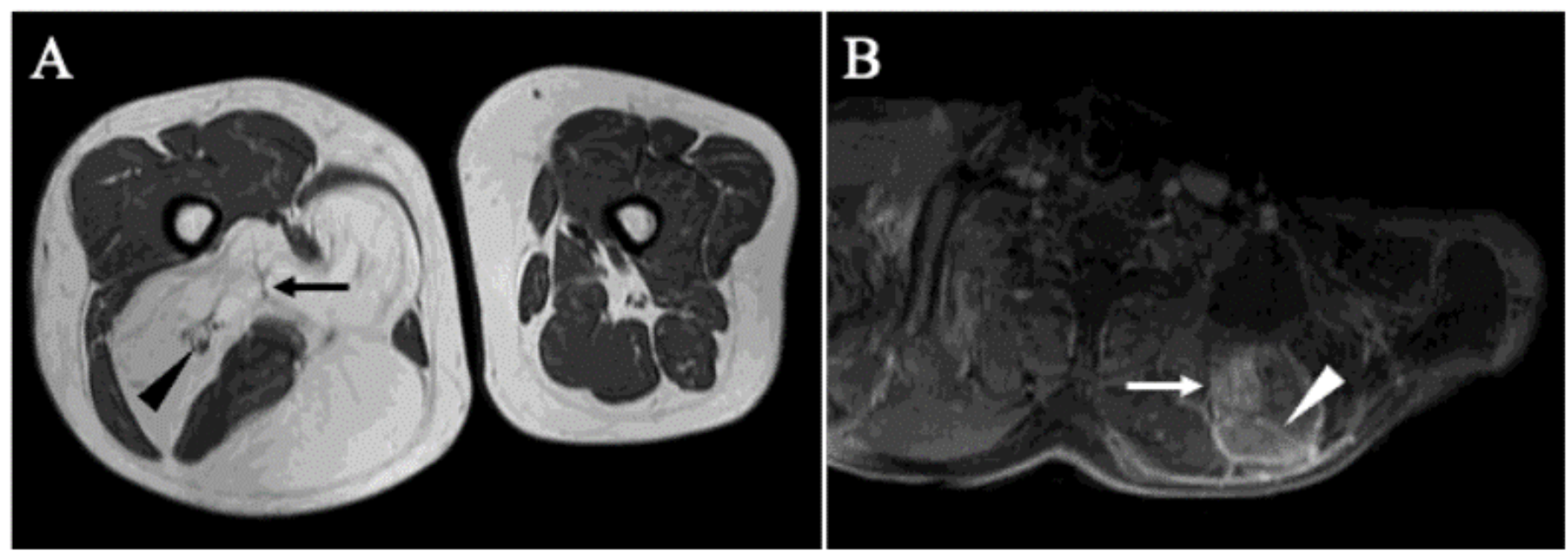

\section{Figure 1}


Radiological findings on MRI used in this study A. Thick septa $(>2 \mathrm{~mm})$ (black arrow) and the sciatic nerve involved in the adipocytic tumor (black triangle) in T1-weighted image. B. Enhancement of septa (white triangle) and nodular lesion (white arrow) in contrast-enhanced fat-suppression T2 image.
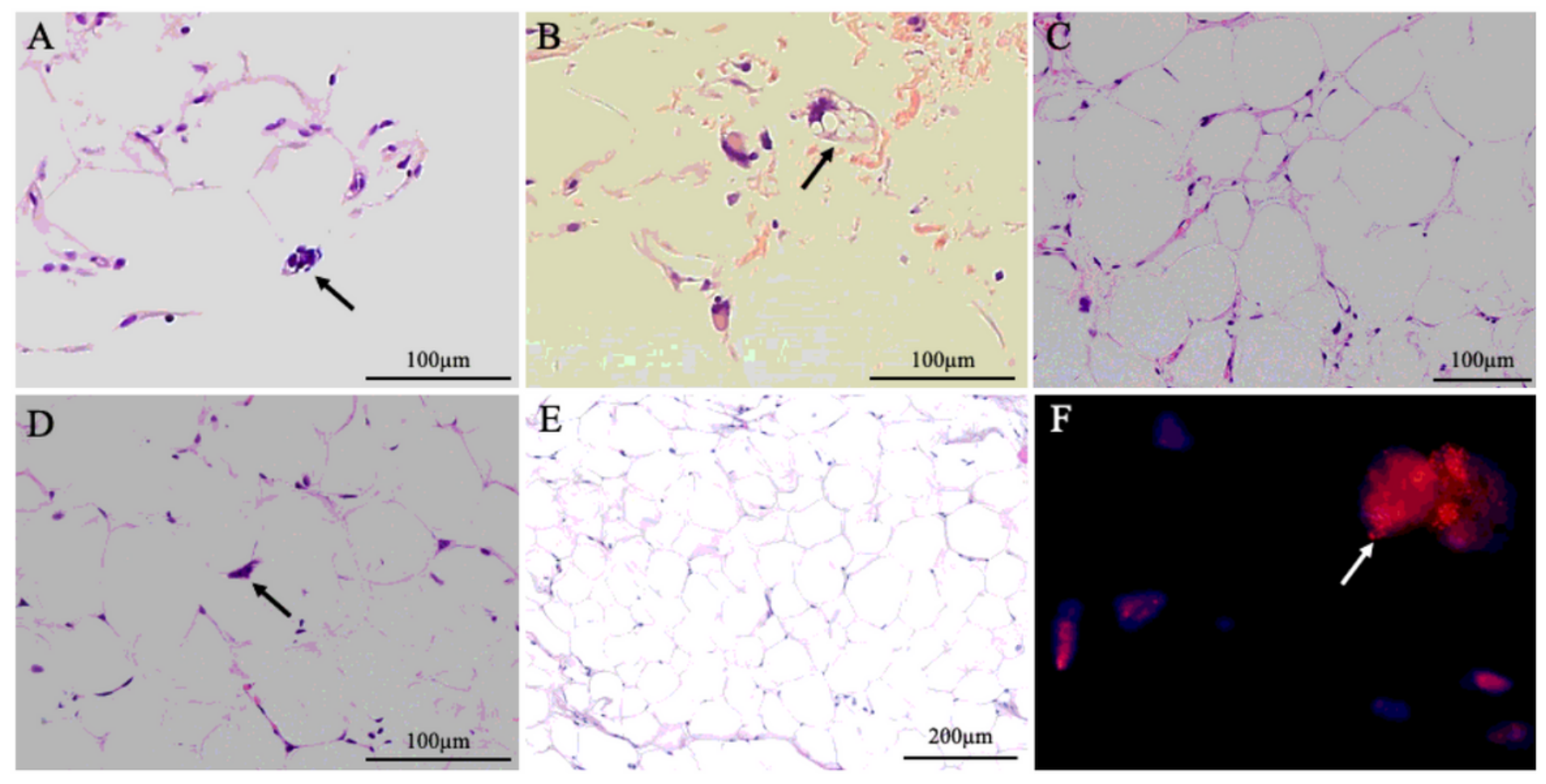

\section{Figure 2}

Histopathological findings by HE staining and FISH examination for MDM2 used in this study (A) Nuclear atypia, (B) lipoblast, (C) differences in size of adipocytes and proliferation of fibrous septa, (D) nuclear enlargement, and (E) lipoma specimen occupied by mature adipocytes without the differences in size in HE staining. (F) MDM2 gene amplification was detected by FISH. 


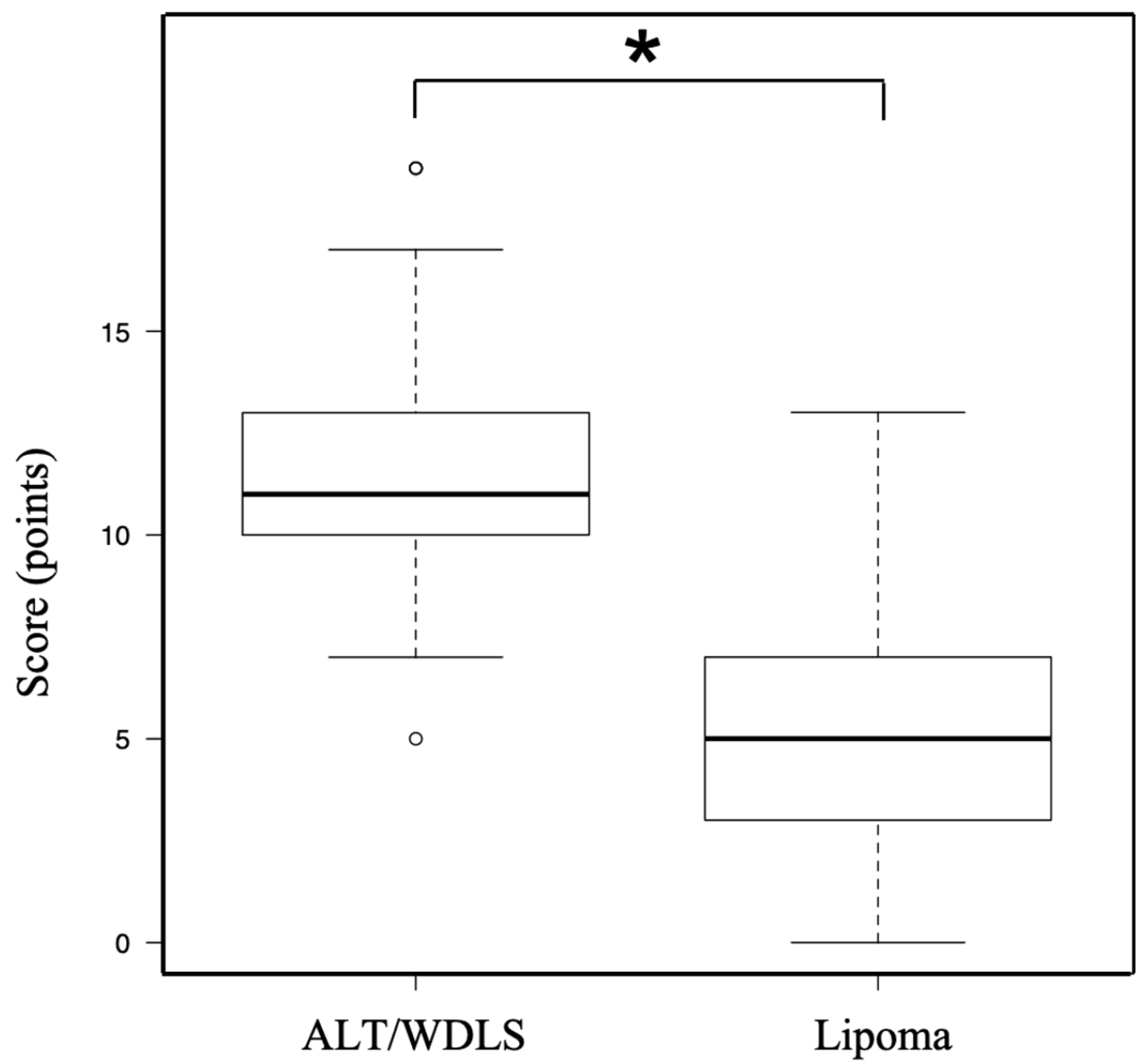

Figure 3

Comparison of the total score of the ALT/WDLS and lipoma groups (mean points were $11.9 \mathrm{vs.} 5.6$, $\left.{ }^{*} p<0.0001\right)$ 